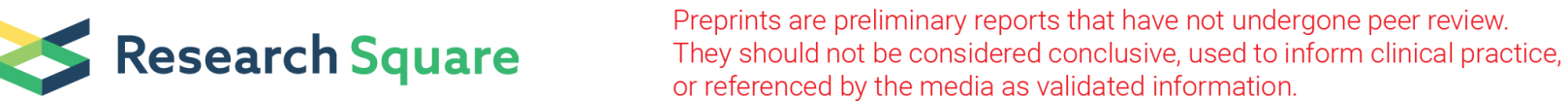

\section{Three and Two-Dimensional Cardiac Mechanic Parameters by Speckle Tracking Echocardiography are Predictors of Outcomes In Chagas Heart Disease}

\section{Viviane Tiemi Hotta ( $\nabla$ viviane.hotta@gmail.com )}

Instituto do Coração (InCor) do Hospital das Clínicas da Faculdade de Medicina da Universidade de São Paulo

\section{Maria Cristina Donadio Abduch}

Instituto do Coração (InCor) do Hospital das Clínicas da Faculdade de Medicina da Universidade de São

Paulo

\section{Marcelo Luiz Campos Vieira}

Instituto do Coração (InCor) do Hospital das Clínicas da Faculdade de Medicina da Universidade de São

Paulo

\section{Andrea Andrade Vilela}

Instituto Dante Pazzanese de Cardiologia

\section{Edimar Alcides Bocchi}

Instituto do Coração (InCor) do Hospital das Clínicas da Faculdade de Medicina da Universidade de São

Paulo

\section{Research Article}

Keywords: Chagas Heart Disease, three-dimensional echocardiography, myocardial strain, cardiac mechanics, outcomes.

Posted Date: November 17th, 2021

DOl: https://doi.org/10.21203/rs.3.rs-1050991/v1

License: (우 (i) This work is licensed under a Creative Commons Attribution 4.0 International License. Read Full License 


\section{Abstract}

BACKGROUND: Chagas disease (CD) is a neglected infectious disease associated with early mortality and substantial disability. Three-dimensional speckle tracking (3D STE) may play a role in the evaluation of CD. We aim to characterize new echocardiographic variables in patients with $C D$ and to assess the hypothesis that 3D STE may predict outcomes.

METHODS: Seventy-two patients with CD were included. Clinical and conventional 2D and 3D STE analysis were performed. Patients were followed up for sixty months. Clinical events were defined as hospitalization for heart failure, complex ventricular arrhythmias, heart transplant and all-cause death.

RESULTS: Seventy-two patients were recruited and enrolled in three groups: left ventricular ejection fraction $(\mathrm{LVEF})<0.40(\mathrm{~N}=22) ; 0.40 \leq \mathrm{LVEF} \leq 0.50(\mathrm{~N}=10)$ and LVEF $>0.50(\mathrm{~N}=30)$. After a Cox model analysis, the top predictors of composite endpoints were 2D LV global longitudinal strain (GLS) $\leq-11.3 \%$ (AUC=0.87), 2D LV global circumferential strain (GCS) $\leq-10.1 \%$ (AUC=0.79), 3D LV GLS $\leq-13 \%$ (AUC=0.82), 3D LV area strain $\leq$ $-16 \%(A U C=0.81)$ and right ventricle (RV) GLS $\leq-17.2 \%(A U C=0.78)$.

CONCLUSIONS: Patients with CD and mrLVEF were morphologically similar to the rLVEF patients despite the benign evolution as the pLVEF group. RV GLS, 2D LV GLS, 2D LV GCS, 3D LV GLS, and 3D LV area strain are strong predictors of sixty months outcomes in patients with $C D$.

\section{Introduction}

Chagas disease (CD) is an endemic and neglected infectious disease, first described in 1909 by Carlos Chagas ${ }^{1}$. Nowadays, more than a century after its description, it still remains associated with early mortality and substantial disability. Nevertheless, in recent decades, CD has increased worldwide and has become a health problem in non-endemic countries as a result of the migration of infected people 2,3 .

CD is characterized by two well established phases: an acute and a chronic phase. Approximately 20 to $40 \%$ of patients with $\mathrm{CD}$ will develop chronic Chagas cardiomyopathy (CCM), which is the most important and severe form of $\mathrm{CD}$. Mortality due to CCM is closely related to the extent of cardiac function impairment and can be attributed to heart failure (HF), conduction disturbances, ventricular arrhythmias and sudden death and/or thromboembolism. CCM carries higher mortality risk than ischemic or idiopathic dilated cardiomyopathies ${ }^{5,6}$.

Despite the crucial importance of an adequate risk profile evaluation for therapeutic guidance, until now, prognostic markers have limited value ${ }^{7,8}$. In this context, echocardiography consists of a very useful tool not only for evaluation of LV function, but also for the analysis of others variables that can add incremental information and provide newer clinical perspectives for patients with $\mathrm{CCM}^{9-14}$.

More recently, techniques as three-dimensional (3D Echo) and speckle tracking echocardiography (STE) have become very useful and promising tools for the evaluation of cardiac mechanics, allowing a more precise and quantitative measurement of the global and regional myocardial longitudinal, radial and circumferential 
strain ${ }^{15,16}$. It is well established in literature that endocardial fibers, arranged longitudinally, are the first to undergo functional damage. Hence, longitudinal myocardial strain has been considered the best predictor of cardiac events in patients with HF, in comparison to left ventricular ejection fraction (LVEF) and Tissue Doppler data ${ }^{15,16}$.

Nevertheless, literature data are scarce concerning cardiac mechanics evaluation by 3D STE, particularly in patients with $\mathrm{CD}$. The present study aims to clinically characterize new echocardiographic variables concerning cardiac mechanics according to LVEF and evaluate its role in the prediction of clinical outcomes in patients with $C D$.

\section{Methods}

Eight hundred and eight patients with CD from the Cardiomyopathies and Heart Failure Clinics of a tertiary cardiological center were evaluated. Seventy-two consecutive patients with CD were recruited based on the following criteria: seropositivity for $\mathrm{CD}$ in two distinct laboratory tests (indirect hemagglutination assay, indirect immunofluorescence technique or ELISA - Enzyme-Linked Immunosorbent Assay) in sinus rhythm without other documented etiology. The exclusion criteria were age under 18 or beyond 70-years old, uncontrolled systemic arterial hypertension, diabetes mellitus, hypothyroidism, renal failure (serum creatinine $>1.5 \mathrm{mg} / \mathrm{dL}$ or glomerular filtration rate $<60 \mathrm{~mL} / \mathrm{min} / 1.73 \mathrm{~m}^{2}$ ), hepatic failure, atrial fibrillation or frequent arrhythmias, coronary artery disease, patients with pacemakers, pregnancy and chronic obstructive pulmonary disease. This was a convenience sample because 3D STE is very sensitive technique and may be influenced by several clinical conditions.

All patients gave written informed consent, and the study was approved by the Hospital das Clínicas, Faculdade de Medicina, Universidade de Sao Paulo Ethical Committee. All patients underwent clinical, electrocardiographic and echocardiographic evaluation. All methods were performed in accordance with the relevant guidelines and regulations.

\section{Echocardiographic Analysis}

All studies were performed using the Vivid E9 (GE Healthcare Medical Systems, Milwaukee, WI, USA) with dedicated transthoracic transducers. Comprehensive conventional (2D Echo) and 3D Echo were performed according to joint recommendations from the American Society of Echocardiography ${ }^{17}$. Two and threedimensional speckle tracking analysis were performed offline using a dedicated software (EchoPAC, BT12, GE Healthcare).

\section{Clinical Follow-up}

All patients were followed up sixty months. All patients were clinically evaluated by an assistant physician every three months or whenever necessary. Holter monitoring was assessed annually for each patient or whenever necessary. Patients were treated according the Brazilian Guidelines for $C D{ }^{5}$. Patients did not receive specific pharmacological treatment for T.cruzi infection. Composite endpoints were defined as 
hospitalization for heart failure (HF), complex ventricular arrhythmias (ventricular fibrillation and sustained ventricular tachycardia), heart transplant and death.

\section{Statistical Analysis}

Continuous numeric variables were expressed as mean \pm standard deviation (SD). Normally distributed data were compared using a 2-sample Student's t-test and 1-way analysis of variance. Non-normally distributed data were compared with Mann-Whitney U test and Kruskal-Wallis test. Categorical variables were expressed as frequency (percentage) and were compared using the chi-square test $\left(x^{2}\right)$ or the Fisher exact test, when appropriate. A two-tailed $p$ value of 0.05 was considered significant.

Intraobserver and interobserver reproducibility of 2D STE parameters were evaluated in 10 randomly selected subjects and evaluated using concordance correlation coefficients (CCC) and Bland-Altman analysis. Intraobserver variability was assessed by having one observer re-measure after 30 days. Another observer blinded to the first observer's measurements evaluated the randomly selected exams for interobserver variability analysis. To determine the optimal cutoff value of prognostic STE parameters for predicting composite endpoints, mortality and hospitalization for heart failure, receiver-operating characteristic (ROC) curves were used.

Survival curves according to the LVEF were obtained in a Kaplan-Meier analysis and compared using the logrank test. Uni and multivariate Cox regression models were use for estimations of the predictors of outcomes.

\section{Results}

Eight-hundred and eight medical electronic reports of patients with CD followed in the single center where the study was performed were analyzed. Seventy-two patients were recruited according to the inclusion and exclusion criteria. The great majority of patients were excluded due to uncontrolled hypertension, diabetes mellitus, irregular cardiac rhythm, hypothyroidism and renal failure. Patients were enrolled in three groups: patients with LVEF $<0.40$ (reduced LVEF, rLVEF) $(\mathrm{N}=32)$; patients with LVEF $\geq 0.40$ and $\leq 0.50$ (mid-range or mildly reduced LVEF, mrLVEF) $(\mathrm{N}=10)$; patients with LVEF > 0.50 (preserved LVEF, pLVEF) $(\mathrm{N}=30)$.

\section{Clinical Characteristics and Conventional Echocardiographic Evaluation}

Clinical, anthropometric and electrocardiographic data are described in Table 1. Conventional 2D Echo variables are comprised in Table 2. There were no difference between the groups regarding gender distribution, mean age and anthropometric variables. 
Table 1

Clinical, anthropometric and electrocardiographic data

\begin{tabular}{|c|c|c|c|c|}
\hline & $\begin{array}{l}\text { LVEF }<0.40 \\
(\mathrm{~N}=32)\end{array}$ & $\begin{array}{l}\text { LVEF } \geq 0.40 \text { and }< \\
0.50 \\
(\mathrm{~N}=10)\end{array}$ & $\begin{array}{l}\text { LVEF } \geq 0.50 \\
(\mathrm{~N}=30)\end{array}$ & p \\
\hline Gender & $\begin{array}{l}\text { Male }=19 \\
(59 \%)\end{array}$ & Male = $7(70 \%)$ & $\begin{array}{l}\text { Male }=14 \\
(47 \%)\end{array}$ & 0,71 \\
\hline Mean Age \pm SD (years) & $53.6 \pm 9,1$ & $48.0 \pm 8.1$ & $54.0 \pm 9.4$ & 0.17 \\
\hline Weight (Kg) & $67.0 \pm 9,9$ & $71.8 \pm 12.0$ & $68.8 \pm 10.9$ & 0.45 \\
\hline Height (cm) & $163.9 \pm 7,7$ & $164.1 \pm 7.5$ & $164.3 \pm 7.2$ & 0.98 \\
\hline $\mathrm{BSA}\left(\mathrm{g} / \mathrm{m}^{2}\right)$ & $1.73 \pm 0.14$ & $1.78 \pm 0.18$ & $1.75 \pm 0.15$ & 0.83 \\
\hline Medical Treatment & $N=18(56 \%)$ & $\mathrm{N}=7(70 \%)$ & $N=5(17 \%)$ & 0.003 \\
\hline ACEi & $\mathrm{N}=11(21 \%)$ & $\mathrm{N}=3(30 \%)$ & $N=5(17 \%)$ & 0.094 \\
\hline ARBs & $N=31(97 \%)$ & $N=10(100 \%)$ & $N=7(23 \%)$ & $<0.001$ \\
\hline Beta Blockers & $N=22(69 \%)$ & $\mathrm{N}=3(30 \%)$ & $N=0$ & $<0.001$ \\
\hline Furosemide & $N=11(21 \%)$ & $N=1(10 \%)$ & $N=3(10 \%)$ & 0.005 \\
\hline Thiazide diuretics & $N=19(59 \%)$ & $\mathrm{N}=7(70 \%)$ & $N=0$ & $<0.001$ \\
\hline Spironolactone & $N=4(13 \%)$ & $N=1(10 \%)$ & $N=0$ & 0.152 \\
\hline Digoxin & $N=5(16 \%)$ & $N=0$ & $N=0$ & 0.002 \\
\hline Nitrates & $N=3(9 \%)$ & $N=0$ & $N=0$ & 0.109 \\
\hline Hydralazine & $N=9(28 \%)$ & $N=1(10 \%)$ & $N=0$ & 0.82 \\
\hline Warfarin & $N=3(9 \%)$ & $N=3(30 \%)$ & $N=2(7 \%)$ & 0.62 \\
\hline \multicolumn{5}{|l|}{ Amiodarone } \\
\hline Functional Class (NYHA) & $N=13(41 \%)$ & $N=5(50 \%)$ & $N=25(83 \%)$ & $<0.001$ \\
\hline$\cdot 1$ & $N=13(41 \%)$ & $\mathrm{N}=4(40 \%)$ & $N=5(17 \%)$ & \\
\hline •॥ & $N=5(16 \%)$ & $\mathrm{N}=1(10 \%)$ & $N=0$ & \\
\hline$\cdot$ •III & $N=1(2 \%)$ & $N=0$ & $N=0$ & \\
\hline \multicolumn{5}{|l|}{$\cdot$ •IV } \\
\hline $\mathrm{SBP}(\mathrm{mmHg})$ & $111.0 \pm 12.3$ & $116.2 \pm 9.8$ & $120.6 \pm 12.7$ & 0.002 \\
\hline
\end{tabular}

* $\mathrm{LVEF}=\mathrm{Left}$ ventricular ejection fraction; $\mathrm{SD}=$ Standard deviation; $\mathrm{BSA}=$ Body surface area; $\mathrm{SAH}=$ Systemic arterial hypertension; $\mathrm{ACE}=$ = Angiotensin-converting-enzyme inhibitors; $\mathrm{ARBs}=$ Angiotensin receptor blockers; ASA = acetylsalicylic acid; NYHA = New York Heart Association; SBP = systolic blood pressure; $\mathrm{DBP}=$ Diastolic blood pressure; $\mathrm{LBBB}=$ Left bundle brunch block; RBBB = Right bundle brunch block; NSVT = Non-sustained ventricular tachycardia; BNP = Brain natriuretic peptide. 


\begin{tabular}{|c|c|c|c|c|}
\hline & $\begin{array}{l}\text { LVEF }<0.40 \\
(\mathrm{~N}=32)\end{array}$ & $\begin{array}{l}\text { LVEF } \geq 0.40 \text { and }< \\
0.50 \\
(\mathrm{~N}=10)\end{array}$ & $\begin{array}{l}\text { LVEF } \geq 0.50 \\
(\mathrm{~N}=30)\end{array}$ & $\mathbf{p}$ \\
\hline $\mathrm{DBP}(\mathrm{mmHg})$ & $71.2 \pm 9.1$ & $77.6 \pm 7.2$ & $78.1 \pm 7.2$ & 0.004 \\
\hline PR interval (ms) & $0.18 \pm 0.06$ & $0.16 \pm 0.03$ & $0.17 \pm 0.02$ & 0.44 \\
\hline QRS interval (ms) & $0.13 \pm 0.03$ & $0.12 \pm 0.03$ & $0.11 \pm 0.03$ & 0.06 \\
\hline Morfology & $\mathrm{N}=4(13 \%)$ & $N=0$ & $\mathrm{~N}=2(7 \%)$ & 0.33 \\
\hline LBBB & $N=12(32 \%)$ & $N=3(30 \%)$ & $N=2(7 \%)$ & 0.42 \\
\hline RBBB & $N=2(6 \%)$ & $N=1(10 \%)$ & $N=5(17 \%)$ & 0.32 \\
\hline \multicolumn{5}{|c|}{$\begin{array}{l}\text { RBBB and left anterior fascicular } \\
\text { block }\end{array}$} \\
\hline Holter & $N=12(38 \%)$ & $N=4(40 \%)$ & $N=1(3 \%)$ & 0.05 \\
\hline \multicolumn{5}{|l|}{ NSVT } \\
\hline Creatinine $(\mathrm{mg} / \mathrm{dl})$ & $1.08 \pm 0.27$ & $0.98 \pm 0.14$ & $0.92 \pm 0.2$ & 0.03 \\
\hline Urea $(\mathrm{mg} / \mathrm{dl})$ & $43.8 \pm 15.6$ & $35.7 \pm 10.2$ & $34.5 \pm 11.4$ & 0.02 \\
\hline \multicolumn{5}{|c|}{$\begin{array}{l}\text { *LVEF = Left ventricular ejection fraction; SD = Standard deviation; BSA = Body surface area; } \mathrm{SAH}= \\
\text { Systemic arterial hypertension; ACEi = Angiotensin-converting-enzyme inhibitors; ARBS = Angiotensin } \\
\text { receptor blockers; ASA = acetylsalicylic acid; NYHA = New York Heart Association; SBP = systolic blood } \\
\text { pressure; DBP = Diastolic blood pressure; LBBB = Left bundle brunch block; RBBB = Right bundle brunch } \\
\text { block; NSVT = Non-sustained ventricular tachycardia; BNP = Brain natriuretic peptide. }\end{array}$} \\
\hline
\end{tabular}


Table 2

Conventional Echocardiographic parameters.

\begin{tabular}{|c|c|c|c|c|}
\hline & $\begin{array}{l}\text { LVEF }<0.40 \\
(\mathrm{~N}=32)\end{array}$ & $\begin{array}{l}\text { LVEF } \geq 0.40 \text { and }< \\
0.50 \\
(\mathrm{~N}=10)\end{array}$ & $\begin{array}{l}\text { LVEF } \geq \\
0.50 \\
(\mathrm{~N}=30)\end{array}$ & p \\
\hline Aorta (mm) & $31.4 \pm 3.4$ & $34.1 \pm 4.1$ & $32.3 \pm 3.2$ & 0.09 \\
\hline Left Atrium (mm) & $43.7 \pm 7.4$ & $40.7 \pm 6.8$ & $37.4 \pm 5.0$ & 0.001 \\
\hline Left atrium volume indexed $\left(\mathrm{ml} / \mathrm{m}^{2}\right)$ & $49.5 \pm 15.9$ & $40.4 \pm 11.2$ & $30.6 \pm 7.6$ & $\begin{array}{l}< \\
0.001\end{array}$ \\
\hline $\mathrm{RA}$ area $\left(\mathrm{cm}^{2}\right)$ & $18.9 \pm 7.4$ & $15.5 \pm 2.5$ & $15.3 \pm 2.7$ & 0.02 \\
\hline RA volume $\left(\mathrm{cm}^{3}\right)$ & $58.2 \pm 39.0$ & $42.2 \pm 10.1$ & $38.1 \pm 12.0$ & 0.01 \\
\hline RV basal diameter (mm) & $37.3 \pm 8.5$ & $32.4 \pm 5.5$ & $32.5 \pm 4.9$ & 0.01 \\
\hline RV mid-level diameter (mm) & $30.0 \pm 7.4$ & $25.8 \pm 5.1$ & $26.7 \pm 4.4$ & 0.04 \\
\hline LVDD (mm) & $66.2 \pm 7.2$ & $59.7 \pm 4.2$ & $49.3 \pm 4.2$ & $\begin{array}{l}< \\
0.001\end{array}$ \\
\hline LVSD (mm) & $59.2 \pm 8.1$ & $49.2 \pm 4.5$ & $33.3 \pm 5.2$ & $\begin{array}{l}< \\
0.001\end{array}$ \\
\hline RWT & $0.24 \pm 0.04$ & $0.27 \pm 0.03$ & $0.34 \pm 0.04$ & $\begin{array}{l}< \\
0.001\end{array}$ \\
\hline Myocardial ass index $\left(\mathrm{g} / \mathrm{m}^{2}\right)$ & $\begin{array}{l}134.9 \pm \\
32.3\end{array}$ & $113.2 \pm 18.3$ & $85.4 \pm 15.3$ & $<.001$ \\
\hline LVDVi (ml/m2) & $\begin{array}{l}117.4 \pm \\
40.4\end{array}$ & $85.4 \pm 22.1$ & $55.0 \pm 16.4$ & $\begin{array}{l}<.001 \\
\text {. }\end{array}$ \\
\hline LVSVi (ml/m2) & $82.7 \pm 32.7$ & $47.2 \pm 14.1$ & $21.2 \pm 7.4$ & $\begin{array}{l}< \\
0.001\end{array}$ \\
\hline LVEF (Simpson method) & $0.31 \pm 0.06$ & $0.45 \pm 0.03$ & $0.62 \pm 0.05$ & $\begin{array}{l}< \\
0.001\end{array}$ \\
\hline TAPSE & $14.7 \pm 3.1$ & $18.0 \pm 4.1$ & $18.4 \pm 2.8$ & $\begin{array}{l}<.001 \\
0.01\end{array}$ \\
\hline RV S' wave & $9.3 \pm 2.2$ & $10.2 \pm 2.9$ & $12.2 \pm 2.0$ & $<.001$ \\
\hline FAC & $0.42 \pm 0.15$ & $0.52 \pm 0.07$ & $0.51 \pm 0.08$ & 0.004 \\
\hline
\end{tabular}

*LVEF = Left ventricular ejection fraction; $S D=$ Standard deviation; $\mathrm{RA}=$ right atrium; $\mathrm{RV}$ = right ventricle; LVDD = Left ventricle diastolic diameter; $L V S D=$ Left ventricle systolic diameter; $R W T$ = relative wall thickness; LVDVi = Left ventricle diastolic volume indexed; LVSDVi = Left ventricle systolic volume indexed; TAPSE = Tricuspid annular plane systolic excursion; FAC = Right ventricle fractional area change; PASP = Pulmonary artery systolic pressure; RT = Tricuspid regurgitation . 


\begin{tabular}{|c|c|c|c|c|}
\hline & $\begin{array}{l}\text { LVEF }<0.40 \\
(\mathrm{~N}=32)\end{array}$ & $\begin{array}{l}\text { LVEF } \geq 0.40 \text { and }< \\
0.50 \\
(\mathrm{~N}=10)\end{array}$ & $\begin{array}{l}\text { LVEF } \geq \\
0.50 \\
(\mathrm{~N}=30)\end{array}$ & $\mathbf{p}$ \\
\hline $\begin{array}{l}\text { Diastolic Function } \\
\text { - Normal } \\
\text { - Grade I } \\
\text { - Grade II } \\
\text { - Grade III } \\
\text { - Grade IV } \\
\text { - Indeterminate }\end{array}$ & $\begin{array}{l}N=0 \\
N=8(25 \%) \\
N=10 \\
(31 \%) \\
N=6(19 \%) \\
N=7(22 \%) \\
N=1(3 \%)\end{array}$ & $\begin{array}{l}N=3(30 \%) \\
N=6(60 \%) \\
N=1(10 \%) \\
N=0 \\
N=0 \\
N=0\end{array}$ & $\begin{array}{l}N=16 \\
(53 \%)\end{array}$ & $\begin{array}{l}< \\
0.001\end{array}$ \\
\hline Septal e' velocity $(\mathrm{cm} / \mathrm{s})$ & $4.9 \pm 1.9$ & $6.0 \pm 2.4$ & $7.9 \pm 1.9$ & $\hat{0}_{0.001}$ \\
\hline Lateral évelocity $(\mathrm{cm} / \mathrm{s})$ & $5.0 \pm 2.3$ & $8.0 \pm 2.9$ & $9.8 \pm 3.2$ & $\hat{0}_{0.001}$ \\
\hline Average $\mathrm{E} / \mathrm{e}^{\prime}$ & $19.7 \pm 12.4$ & $10.2 \pm 4.2$ & $7.9 \pm 2.0$ & $\begin{array}{l}< \\
0.001\end{array}$ \\
\hline
\end{tabular}

*LVEF = Left ventricular ejection fraction; $S D=$ Standard deviation; $\mathrm{RA}=$ right atrium; $\mathrm{RV}=$ right ventricle; LVDD = Left ventricle diastolic diameter; $L V S D=$ Left ventricle systolic diameter; $R W T$ = relative wall thickness; LVDVi = Left ventricle diastolic volume indexed; LVSDVi = Left ventricle systolic volume indexed; TAPSE = Tricuspid annular plane systolic excursion; FAC = Right ventricle fractional area change; PASP = Pulmonary artery systolic pressure; RT = Tricuspid regurgitation . 


\begin{tabular}{|c|c|c|c|c|}
\hline & $\begin{array}{l}\text { LVEF }<0.40 \\
(\mathrm{~N}=32)\end{array}$ & $\begin{array}{l}\text { LVEF } \geq 0.40 \text { and }< \\
0.50 \\
(N=10)\end{array}$ & $\begin{array}{l}\text { LVEF } \geq \\
0.50 \\
(\mathrm{~N}=30)\end{array}$ & p \\
\hline \multirow{5}{*}{$\begin{array}{l}\text { Mitral regurgitation } \\
\text { - Absent } \\
\text { - Mild }\end{array}$} & \multirow{3}{*}{$\begin{array}{l}N=5(16 \%) \\
N=11 \\
(34 \%)\end{array}$} & $N=2(20 \%)$ & $\begin{array}{l}N= \\
23(77 \%)\end{array}$ & \multirow{5}{*}{$\begin{array}{l}<.001 \\
0.00\end{array}$} \\
\hline & & \multirow{4}{*}{$\begin{array}{l}N=4(40 \%) \\
N=3(30 \%)\end{array}$} & & \\
\hline & & & (2) & \\
\hline & $N=7(22 \%)$ & & $N=0$ & \\
\hline & \multirow[t]{3}{*}{$N=9(28 \%)$} & & $N=0$ & \\
\hline - Moderate & & $N=1(10 \%)$ & & \\
\hline - Severe & & & & \\
\hline \multirow{5}{*}{$\begin{array}{l}\text { Aortic regurgitation } \\
\text { - Absent } \\
\text { - Mild } \\
\text { - Moderate } \\
\text { - Severe }\end{array}$} & \multirow{5}{*}{$\begin{array}{l}N=26 \\
(81 \%) \\
N=6(19 \%) \\
N=0 \\
N=0\end{array}$} & $N=7(70 \%)$ & $N=26$ & \multirow[t]{5}{*}{0.28} \\
\hline & & $N=3(30 \%)$ & $N=2$ & \\
\hline & & $N=0$ & $N=0$ & \\
\hline & & \multirow[t]{2}{*}{$N=0$} & $N=0$ & \\
\hline & & & & \\
\hline \multirow{5}{*}{$\begin{array}{l}\text { Tricuspid regurgitation } \\
\cdot \text { Absent } \\
\text { - Mild } \\
\text { - Moderate } \\
\text { - Severe }\end{array}$} & \multirow{2}{*}{$\begin{array}{l}N=25 \\
(78 \%)\end{array}$} & \multirow{5}{*}{$\begin{array}{l}N=10(100 \%) \\
N=0 \\
N=0 \\
N=0\end{array}$} & $\begin{array}{l}N=24 \\
(86 \%)\end{array}$ & \multirow[t]{5}{*}{0.06} \\
\hline & & & $\mathrm{N}=4(14 \%)$ & \\
\hline & \multirow{3}{*}{$\begin{array}{l}N=2(6 \%) \\
N=1(3 \%) \\
N=4(13 \%)\end{array}$} & & $N=0$ & \\
\hline & & & $N=0$ & \\
\hline & & & & \\
\hline PASP (mmHg) & $51.4 \pm 14.4$ & NA & $27.5 \pm 7.5$ & 0.02 \\
\hline TR peak velocity $(\mathrm{m} / \mathrm{s})$ & $3.36 \pm 0.40$ & NA & $2.36 \pm 0.28$ & 0.03 \\
\hline
\end{tabular}

*LVEF = Left ventricular ejection fraction; $S D=$ Standard deviation; $\mathrm{RA}=$ right atrium; $\mathrm{RV}$ = right ventricle; LVDD = Left ventricle diastolic diameter; $L V S D=$ Left ventricle systolic diameter; $R W T$ = relative wall thickness; $L V D V i=$ Left ventricle diastolic volume indexed; LVSDVi = Left ventricle systolic volume indexed; TAPSE = Tricuspid annular plane systolic excursion; FAC = Right ventricle fractional area change; PASP = Pulmonary artery systolic pressure; RT = Tricuspid regurgitation . 


\begin{tabular}{|c|c|c|c|c|}
\hline & $\begin{array}{l}\text { LVEF }<0.40 \\
(\mathrm{~N}=32)\end{array}$ & $\begin{array}{l}\text { LVEF } \geq 0.40 \text { and }< \\
0.50 \\
(\mathrm{~N}=10)\end{array}$ & $\begin{array}{l}\text { LVEF } \geq \\
0.50 \\
(\mathrm{~N}=30)\end{array}$ & $\mathrm{p}$ \\
\hline \multirow{3}{*}{$\begin{array}{l}\text { Diffuse hypokinesis } \\
\text { Inferior and lateral inferior walls } \\
\text { akinesis }\end{array}$} & $\begin{array}{l}N=25 \\
(78 \%)\end{array}$ & $N=3(30 \%)$ & $\mathrm{N}=0$ & \multirow[t]{8}{*}{0.02} \\
\hline & \multirow{2}{*}{$\mathrm{N}=3(9 \%)$} & \multirow{5}{*}{$\begin{array}{l}N=3(30 \%) \\
N=0\end{array}$} & $\mathrm{~N}=2(7 \%)$ & \\
\hline & & & $N=0$ & \\
\hline Lateral inferior wall akinesis & $\mathrm{N}=1(3 \%)$ & & & \\
\hline Apical akinesis & $N=2(6 \%)$ & & & \\
\hline \multirow[t]{3}{*}{ Apical Aneurysm } & \multirow[t]{3}{*}{$N=1(3 \%)$} & & & \\
\hline & & $N=2(20 \%)$ & $\mathrm{N}=0$ & \\
\hline & & $\mathrm{N}=2(20 \%)$ & $\mathrm{N}=0$ & \\
\hline Apical thrombus & $N=1(3 \%)$ & $N=0$ & $N=0$ & 0.36 \\
\hline \multirow{2}{*}{$\begin{array}{l}\text { Pericardial Effusion } \\
\text { - Absent } \\
\text { • Mild }\end{array}$} & $\begin{array}{l}N=30 \\
(94 \%)\end{array}$ & $\mathrm{N}=9(90 \%)$ & $\begin{array}{l}N=27 \\
(95 \%)\end{array}$ & \multirow[t]{2}{*}{0.67} \\
\hline & $N=2(6 \%)$ & $\mathrm{N}=1(10 \%)$ & $N=1(5 \%)$ & \\
\hline $\begin{array}{l}\text { *LVEF = Left ventricular ejection } \\
\text { LVDD = Left ventricle diastolic di } \\
\text { thickness; LVDVi = Left ventricle } \\
\text { indexed; TAPSE = Tricuspid ann } \\
\text { PASP = Pulmonary artery systoli }\end{array}$ & $\begin{array}{l}\text { SD }=\text { Standar } \\
\text { VSD = Left v } \\
\text { volume index } \\
\text { e systolic excL } \\
\text { re; RT = Tricus }\end{array}$ & $\begin{array}{l}\text { deviation; RA = right } \\
\text { tricle systolic diame } \\
\text { d; LVSDVi = Left vent } \\
\text { sion; FAC = Right ven } \\
\text { id regurgitation. }\end{array}$ & $\begin{array}{l}\mathrm{m} ; \mathrm{RV}=\text { righ } \\
\mathrm{WT}=\text { relativ } \\
\text { systolic vol } \\
\text { fractional a }\end{array}$ & $\begin{array}{l}\text { ntricle; } \\
\text { all } \\
\text { change; }\end{array}$ \\
\hline
\end{tabular}

Bilateral cardiac chambers dimensions were larger in the rLVEF in comparison to the group with pLVEF. LV diastolic and systolic indexed volumes were greater in the group rLVEF than in the other groups. Thus, in these patients, LV indexed volumes were more accurate to distinguish the groups according to the LVEF than LV diameters and absolute volumes. Tricuspid annular plane systolic excursion (TAPSE) was the only RV systolic function parameter able to distinguish the groups. Septal e' and lateral e' velocities on Tissue Doppler were lower while E/e' ratios were greater in the group rLVEF in G1 when compared to the other groups.

\section{Two-dimensional STE (2D STE)}

2D STE data are shown in Table 3. 2D STE feasibility was very high in all the groups. LV GLS values were able to distinguish the groups according to the LVEF. RV GLS and RV free wall longitudinal strain values were lower in the group rLVEF in comparison to the other groups. 
Table 3

Echocardiographic parameters of myocardial longitudinal, radial, circumferential strain and displacement evaluated by $2 \mathrm{D}$ STE.

\begin{tabular}{|c|c|c|c|c|}
\hline & $\begin{array}{l}\text { LVEF }<0.40 \\
(\mathrm{rLVEF}) \\
(\mathrm{N}=32)\end{array}$ & $\begin{array}{l}\text { LVEF } \geq 0.40 \text { and }<0.50 \\
(\mathrm{mrLVEF}) \\
(\mathrm{N}=10)\end{array}$ & $\begin{array}{l}\text { LVEF } \geq 0.50 \\
(\mathrm{pLVEF}) \\
(\mathrm{N}=30)\end{array}$ & $\mathbf{p}$ \\
\hline Heart rate $(\mathrm{bpm})$ & $62.3 \pm 11.4$ & $64.6 \pm 12.0$ & $62.2 \pm 11.8$ & 0.62 \\
\hline Frame Rate (qps) & $59.9 \pm 7.4$ & $58.2 \pm 5.1$ & $60.4 \pm 8.9$ & 0.77 \\
\hline Exequibility & $\begin{array}{l}N=572 / 576 \\
(99.3 \%)\end{array}$ & $N=178 / 180(98.9 \%)$ & $\begin{array}{l}N=504 / 504 \\
(100 \%)\end{array}$ & 0.20 \\
\hline $\begin{array}{l}\text { LV Global Longitudinal } \\
\text { Strain }\end{array}$ & $-9.2 \pm 2.8$ & $-14.0 \pm 3.4$ & $-19.1 \pm 3.6$ & $\begin{array}{l}<0.001 * \\
* *,+\end{array}$ \\
\hline $\begin{array}{l}\text { Apical 3-chambers } \\
\text { GLS (\%) }\end{array}$ & $-9.5 \pm 3.5$ & $-13.9 \pm 3.2$ & $-20.1 \pm 4.1$ & $\begin{array}{l}<0.001 * * \\
\dagger\end{array}$ \\
\hline $\begin{array}{l}\text { Apical 4-chambers } \\
\text { GLS (\%) }\end{array}$ & $-8.5 \pm 3.3$ & $-13.4 \pm 3.4$ & $-19.1 \pm 4.2$ & $\begin{array}{l}<0.001 * * \\
\dagger\end{array}$ \\
\hline $\begin{array}{l}\text { Apical 2-chambers } \\
\text { GLS (\%) }\end{array}$ & $-9.2 \pm 3.3$ & $-13.3 \pm 4.2$ & $-16.1 \pm 7.8$ & $<0.001 *$ \\
\hline \multirow{2}{*}{$\begin{array}{l}\text { Basal (Papillary } \\
\text { muscles) } \\
\text { CS Systolic Peak (\%) }\end{array}$} & $-7.3 \pm 2.1$ & $-9.2 \pm 1.9$ & $-12.3 \pm 2.1$ & $<0.001$ **, \\
\hline & $11.0 \pm 6.5$ & $13.9 \pm 9.6$ & $\begin{array}{l}15.3 \pm 9.1 \\
5.0 \pm 2.6\end{array}$ & \multirow{2}{*}{$\begin{array}{l}0.12 \\
0.002 * \text {,** }\end{array}$} \\
\hline \multirow{2}{*}{ Displacement (\%) } & $-7.5 \pm 2.4$ & $-7.1 \pm 4.3$ & $-11.5 \pm 3.2$ & \\
\hline & $13.4 \pm 9.2$ & $14.4 \pm 6.0$ & $28.7 \pm 14.7$ & $\begin{array}{l}<0.001 \text { **, } \\
+\end{array}$ \\
\hline $\begin{array}{l}\text { Mid (Mitral valve) } \\
\text { CS Systolic Peak (\%) }\end{array}$ & $3.3 \pm 1.1$ & $3.9 \pm 0.8$ & $\begin{array}{l}5.1 \pm 1.3 \\
-15.0 \pm 3.8\end{array}$ & $\begin{array}{l}<0.001 * *, \\
\dagger\end{array}$ \\
\hline RS (\%) & $11.1 \pm 7.6$ & $15.5 \pm 8.1$ & $25.6 \pm 20.8$ & $\begin{array}{l}<0.001 \text { ** } \\
+\end{array}$ \\
\hline \multirow{2}{*}{$\begin{array}{l}\text { Displacement (\%) } \\
\text { Apical } \\
\text { CS Systolic Peak (\%) }\end{array}$} & $2.5 \pm 1.3$ & $3.1 \pm 0.9$ & $4.2 \pm 1.0$ & \multirow{2}{*}{$\begin{array}{l}0.003 * *,+ \\
0.001 * *, \dagger \\
<0.001 * * \\
+\end{array}$} \\
\hline & & & & \\
\hline \multicolumn{5}{|l|}{ Displacement (\%) } \\
\hline RV GLS & $-15.6 \pm 5.2$ & $-21,5 \pm 2.6$ & $-21 \pm 3.4$ & $<0.001 *$ \\
\hline $\begin{array}{l}{ }^{*} \mathrm{LV}=\text { Left ventricle; } \mathrm{RV} \\
\text { Radial strain; } \mathrm{FW}=\text { free } \\
0.05 . \mathrm{rLVEF} \text {, reduced left } \\
\text { preserved left ventricula }\end{array}$ & $\begin{array}{l}\text { ight ventricle } \\
\text { all. * rLVEF x } \\
\text { entricular eje }\end{array}$ & $\begin{array}{l}\text { Global longitudinal strair } \\
\text { F, p< } 0.05,{ }^{*} \text { rLVEF x pLV } \\
\text { action; mrLVEF, mid-rang }\end{array}$ & $\begin{array}{l}\text { Circumferent } \\
<0.05,+\mathrm{mrL} \\
\text { mildly ejectior }\end{array}$ & $\begin{array}{l}\text { ain; RS: } \\
\text { pLVEF, p< } \\
\text { tion; pLVEF, }\end{array}$ \\
\hline
\end{tabular}




\begin{tabular}{|lllll|}
\hline & $\begin{array}{l}\text { LVEF }<0.40 \\
(\mathrm{ILVEF}) \\
(\mathrm{N}=32)\end{array}$ & $\begin{array}{l}\text { LVEF } \geq 0.40 \text { and }<0.50 \\
(\mathrm{mrLVEF}) \\
(\mathrm{N}=10)\end{array}$ & $\begin{array}{l}\text { LVEF } \geq 0.50 \\
(\mathrm{pLVEF})\end{array}$ & $\mathrm{p}$ \\
$\mathbf{( N = 3 0 )}$ & $-24.1 \pm 4.4$ & $<0.001 *$ \\
R*
\end{tabular}

LV Peak systolic CS and LV displacement were lower in the group rLVEF in comparison to the group pLVEF. With exception of LV basal displacement, there were no differences regarding LV peak systolic CS and displacement between the groups rLVEF and mrLVEF. RV GLS and free wall strain were similar between the groups with mrLVEF and pLVEF.

\section{Three-dimensional Echocardiographic results}

3D Conventional Echo and STE parameters are shown in Table 4. There were no statistical differences between rLVEF and mrLVEF regarding left atrium (LA) indexed volume, LV end systolic indexed volume, sphericity index. 3D STE feasibility was very good in all the groups. GLS was different between the groups. There were no statistical differences between mrLVEF and pLVEF regarding 3D LV GLS, LV GCS, LV GCS or LV area strain. Figure 1 depicts 3D LV GLS, LV GRS, LV GCS and LV area strain of a patient with CD and severe LV dysfunction. 
Table 4

Conventional and STE parameters by 3D Echo according to the LVEF.

\begin{tabular}{|c|c|c|c|c|}
\hline & $\begin{array}{l}\text { LVEF }<0.40 \\
(r L V E F) \\
(N=32)\end{array}$ & $\begin{array}{l}\text { LVEF } \geq 0.40 \text { and }< \\
0.50 \\
(\mathrm{mrLVEF}) \\
(\mathrm{N}=10)\end{array}$ & $\begin{array}{l}\text { LVEF } \geq 0.50 \\
(\mathrm{pLVEF}) \\
(\mathrm{N}=30)\end{array}$ & p \\
\hline LA indexed volume $\left(\mathrm{ml} / \mathrm{m}^{2}\right)$ & $37.6 \pm 13.6$ & $31.1 \pm 10.1$ & $21.3 \pm 7.2$ & $\begin{array}{l}<0.001 * * \\
\dagger\end{array}$ \\
\hline LVEDVi (ml) & $114.3 \pm 35.8$ & $92.2 \pm 16.5$ & $58.0 \pm 26.5$ & $\underset{\dagger}{<0.001 * *}$ \\
\hline LVESVi (ml) & $79.5 \pm 28.3$ & $50.6 \pm 12.0$ & $39.2 \pm 10.5$ & $\begin{array}{l}<0.001 * \\
* \star,+\end{array}$ \\
\hline LVEF (\%) & $0.31 \pm 0.06$ & $0.46 \pm 0.05$ & $0.61 \pm 0.03$ & $\begin{array}{l}<0.001 * \\
* \star,+\end{array}$ \\
\hline Sphericity index & $0.48 \pm 0.12$ & $0.43 \pm 0.08$ & $0.36 \pm 0.08$ & $\underset{\dagger}{<0.001 * *}$ \\
\hline $\begin{array}{l}\text { LV myocardial mass index } \\
\left(\mathrm{g} / \mathrm{m}^{2}\right)\end{array}$ & $157.8 \pm 50.9$ & $139.3 \pm 35.5$ & $108.0 \pm 27.5$ & $\begin{array}{l}<0.001 * * \\
+\end{array}$ \\
\hline Exequibility 3D STE & $\begin{array}{l}N=527 / 576 \\
(91.5 \%)\end{array}$ & $\begin{array}{l}N=161 / 180 \\
(89.4 \%)\end{array}$ & $\begin{array}{l}N=421 / 476 \\
(88 \%)\end{array}$ & 0.83 \\
\hline $\begin{array}{l}\text { Global Longitudinal Strain } \\
(\%)\end{array}$ & $-9.0 \pm 5.1$ & $-14.7 \pm 3.7$ & $-16.8 \pm 3.3$ & $0.001 *, * *$ \\
\hline $\begin{array}{l}\text { Global Circunferencial } \\
\text { Strain (\%) }\end{array}$ & $-9.1 \pm 3.9$ & $-12.5 \pm 3.6$ & $-14.5 \pm 6.9$ & $0.001 *, * *$ \\
\hline Global Radial Strain (\%) & $21.8 \pm 11.0$ & $37.6 \pm 16.2$ & $46.5 \pm 11.5$ & $<0.001 *$ \\
\hline Área Strain (\%) & $-15.5 \pm 6.8$ & $-24.6 \pm 5.7$ & $-28.0 \pm 5.2$ & $<0.001 *$ \\
\hline $\begin{array}{l}\text { * } \mathrm{LA}=\mathrm{Left} \text { atrium; } \mathrm{LVEF}=\mathrm{Lef} \\
\text { volume; } \mathrm{LVESVi}=\text { Indexed } \mathrm{Le} \\
\mathrm{p}<0.05,+\mathrm{mrLVEF} \times \mathrm{pLVEF}, \mathrm{p} \\
\text { or mildly ejection fraction; } p \mathrm{~L}\end{array}$ & $\begin{array}{l}\text { ventricle ejectic } \\
\text { ventricle end s } \\
0.05 \text {. rLVEF, re } \\
\text { EF, preserved le }\end{array}$ & $\begin{array}{l}\text { tion; LVEDVi = Index } \\
\text { c volume. * rLVEF x n } \\
\text { left ventricular eject } \\
\text { tricular ejection fract }\end{array}$ & $\begin{array}{l}\text { Left ventricle } \\
\text { VEF, } p<0.05 \text {, * } \\
\text { fraction; } \mathrm{mrL}\end{array}$ & $\begin{array}{l}\text { diastolic } \\
\text { 'EF x pLVEF, } \\
\text { mid-range }\end{array}$ \\
\hline
\end{tabular}

\section{Clinical Follow up}

All patients were followed up for sixty months. Composite endpoints (hospitalization for HF, complex ventricular arrhythmias, heart transplant and death), survival and hospitalization for heart failure Kaplan Meyer curves are shown in Figure 2. One patient in PLVEF died of non-cardiovascular cause. There were no clinical events in mrLVEF. There were nine hospitalizations for decompensated HF, two episodes of complex ventricular arrhythmias, two heart transplants and seventeen deaths in rLVEF. 


\section{Composite Endpoints}

In the overall patients, bilateral cardiac remodeling was associated with composite endpoints. Larger atrial and ventricular diameters and volumes (absolute or indexed) as well as reduced biventricular function evaluated by 2D and 3D Echo, reduced velocities at tissue Doppler and increased E/e' ratio were associated with composite endpoints. 2D LV GLS, 2D LV GCS, RV GLS, 3D LV GLS and 3D LV area strain were the strongest predictors of composite endpoints (Table 5). 
Table 5

Predictors of Composite Endpoints, overall mortality and hospitalization for heart failure in patients with Chagas Disease

\begin{tabular}{|c|c|c|c|c|c|c|c|c|c|}
\hline \multirow[b]{2}{*}{ Variable } & \multicolumn{3}{|c|}{$\begin{array}{l}\text { Hospitalization for Heart } \\
\text { Failure }\end{array}$} & \multicolumn{3}{|l|}{ Death } & \multicolumn{3}{|c|}{ Composite Endpoints } \\
\hline & $\mathbf{p}$ & HR & $95 \% \mathrm{Cl}$ & p & HR & $\begin{array}{l}95 \% \\
\mathrm{Cl}\end{array}$ & p & HR & $\begin{array}{l}95 \% \\
\mathrm{Cl}\end{array}$ \\
\hline LA Diameter & 0.002 & 1.188 & $1.063-1.328$ & 0.015 & 1.093 & $\begin{array}{l}1.018- \\
1.174\end{array}$ & $<0.001$ & 1,121 & $\begin{array}{l}1,055- \\
1,192\end{array}$ \\
\hline LA volume & $<0.001$ & 1.060 & $1.030-1.092$ & $<0.001$ & 1.026 & $\begin{array}{l}1.011- \\
1.041\end{array}$ & $<0.001$ & 1,027 & $\begin{array}{l}1,015- \\
1,040\end{array}$ \\
\hline $\begin{array}{l}\text { LA indexed } \\
\text { volume } \\
\left(\mathrm{ml} / \mathrm{m}^{2}\right)\end{array}$ & $<0.001$ & 1.061 & $1.03-1.092$ & $<0.001$ & 1.052 & $\begin{array}{l}1.025- \\
1.087\end{array}$ & $<0.001$ & 1,05 & $\begin{array}{l}1,028- \\
1,072\end{array}$ \\
\hline $\begin{array}{l}\text { RA area } \\
\left(\mathrm{cm}^{2}\right)\end{array}$ & $<0.001$ & 1.183 & $1.086-1.289$ & $<0.001$ & 1.144 & $\begin{array}{l}1.070- \\
1.224\end{array}$ & $<0.001$ & 1,206 & $\begin{array}{l}1,12- \\
1,29\end{array}$ \\
\hline $\begin{array}{l}\text { RA volume } \\
(\mathrm{ml})\end{array}$ & $<0.001$ & 1.030 & $1.015-1.045$ & $<0.001$ & 1.026 & $\begin{array}{l}1.013- \\
1.039\end{array}$ & $<0.001$ & 1,036 & $\begin{array}{l}1,022- \\
1,049\end{array}$ \\
\hline $\begin{array}{l}\text { TAPSE } \\
(\mathrm{mm})\end{array}$ & 0.12 & 0.853 & $0.699-1.041$ & 0.02 & 0.850 & $\begin{array}{l}0.740- \\
0.976\end{array}$ & 0.009 & 0,854 & $\begin{array}{l}0,76- \\
0,96\end{array}$ \\
\hline $\begin{array}{l}\mathrm{RV} \mathrm{S}^{\prime} \text { wave } \\
(\mathrm{cm} / \mathrm{s})\end{array}$ & 0.006 & 0.682 & $0.521-0.894$ & 0.05 & 0.823 & $\begin{array}{l}0.678- \\
1.000\end{array}$ & 0.003 & 0,784 & $\begin{array}{l}0,669- \\
0,92\end{array}$ \\
\hline FAC & 0.002 & 0.001 & $0.000-0.064$ & 0.06 & 0.040 & $\begin{array}{l}0.001- \\
1.070\end{array}$ & 0.001 & 0,008 & $\begin{array}{l}0,001- \\
0,128\end{array}$ \\
\hline LVDD (mm) & $<0.001$ & 1.147 & $1.063-1.238$ & $<0.001$ & 1.123 & $\begin{array}{l}1.063- \\
1.186\end{array}$ & $<0.001$ & 1,167 & $\begin{array}{l}1,107- \\
1,229\end{array}$ \\
\hline LVSD (mm) & 0.001 & 1.118 & $1.044-1.197$ & $<0.001$ & 1.104 & $\begin{array}{l}1.055- \\
1.156\end{array}$ & $<0.001$ & 1,123 & $\begin{array}{l}1,077- \\
1,171\end{array}$ \\
\hline RWT & 0.001 & 0.000 & $0.000-0.000$ & $<0.001$ & 0.000 & $\begin{array}{l}0.000- \\
0.003\end{array}$ & $<0.001$ & 0.000 & $\begin{array}{l}0,00- \\
0,00\end{array}$ \\
\hline LVEDV (ml) & 0.001 & 1.011 & $1.005-1.017$ & $<0.001$ & 1.011 & $\begin{array}{l}1.005- \\
1.016\end{array}$ & $<0.001$ & 1,013 & $\begin{array}{l}1,009- \\
1,017\end{array}$ \\
\hline LVESV (ml) & $<0.001$ & 1.013 & $1.006-1.021$ & $<0.001$ & 1.013 & $\begin{array}{l}1.007- \\
1.019\end{array}$ & $<0.001$ & 1,016 & $\begin{array}{l}1,011- \\
1,021\end{array}$ \\
\hline
\end{tabular}

$\mathrm{HR}=$ hazard ratio; $\mathrm{Cl}=$ confidence interval). ${ }^{*}$ Values of $\mathrm{p}<0.05$ indicate statistical significance.

FAC, fractional area change; LA, left atrium; LV, left ventricle; LVDD, left ventricular diastolic diameter; LVDV, left ventricular diastolic volume; LVEDV, left ventricular end-diastolic volume; LVEDV, left ventricular end-systolic volume; LVEF, left ventricular ejection fraction; LVSD, left ventricular systolic diameter; LVDV, left ventricular diastolic diameter; RA, right atrium; RV, right ventricle; RWT, relative wall thickness; TAPSE, tricuspid annular plane systolic excursion. 


\section{Hospitalization for Heart \\ Failure}

\begin{tabular}{|c|c|c|c|c|c|c|c|c|c|}
\hline $\begin{array}{l}\text { Indexed } \\
\text { Myocardial } \\
\text { Mass } \\
\left(\mathrm{g} / \mathrm{m}^{2}\right)\end{array}$ & 0.025 & 1.021 & $1.003-1.039$ & $<0.001$ & 1.026 & $\begin{array}{l}1.013- \\
1.040\end{array}$ & $<0.001$ & 1,027 & $\begin{array}{l}1,016- \\
1,038\end{array}$ \\
\hline $\begin{array}{l}\text { LVEF } \\
\text { (Simpson) }\end{array}$ & 0.004 & 0.000 & $0.000-0.031$ & $<0.001$ & 0.000 & $\begin{array}{l}0.000- \\
0.014\end{array}$ & $<0.001$ & 0.000 & $\begin{array}{l}0,000- \\
0,003\end{array}$ \\
\hline $\begin{array}{l}\text { LVDV } \\
\text { indexed (ml) }\end{array}$ & 0.002 & 1.017 & $1.007-1.029$ & $<0.001$ & 1.020 & $\begin{array}{l}1.010- \\
1.030\end{array}$ & $<0.001$ & 1,021 & $\begin{array}{l}1,014- \\
1,029\end{array}$ \\
\hline $\begin{array}{l}\text { LVSV } \\
\text { indexed (ml) }\end{array}$ & $<0.001$ & 1.022 & $1.010-1.034$ & $<0.001$ & 1.023 & $\begin{array}{l}1.012- \\
1.034\end{array}$ & $<0.001$ & 1,026 & $\begin{array}{l}1,017- \\
1,035\end{array}$ \\
\hline $\begin{array}{l}\text { E wave } \\
(\mathrm{cm} / \mathrm{s})\end{array}$ & 0.001 & 1.051 & $1.02-1.084$ & 0.002 & 1.038 & 1.013 & $<0.001$ & 1,038 & $\begin{array}{l}1,018- \\
1,059\end{array}$ \\
\hline $\begin{array}{l}\text { A wave } \\
(\mathrm{cm} / \mathrm{s})\end{array}$ & 0.032 & 0.942 & 0.893.0.995 & 0.06 & 0.972 & $\begin{array}{l}0.943- \\
1.001\end{array}$ & 0.021 & 0,97 & $\begin{array}{l}0,945- \\
0,995\end{array}$ \\
\hline $\begin{array}{l}\text { Septal e' } \\
(\mathrm{cm} / \mathrm{s})\end{array}$ & 0.021 & 0.65 & $0.451-0.937$ & 0.002 & 0.692 & $\begin{array}{l}0.546- \\
0.875\end{array}$ & 0.001 & 0,713 & $\begin{array}{l}0,587- \\
0,867\end{array}$ \\
\hline $\begin{array}{l}\text { Lateral e' } \\
(\mathrm{cm} / \mathrm{s})\end{array}$ & 0.018 & 0.732 & $0.564-0.948$ & $<0.001$ & 0.694 & $\begin{array}{l}0.574- \\
0.839\end{array}$ & $<0.001$ & 0,714 & $\begin{array}{l}0,609- \\
0,836\end{array}$ \\
\hline $\begin{array}{l}\text { Mean E/e } \\
\text { 'ratio }\end{array}$ & $<0.001$ & 1.076 & 1.035-1.119 & $<0.001$ & 1.055 & $\begin{array}{l}1.025- \\
1.085\end{array}$ & $<0.001$ & 1,059 & $\begin{array}{l}1,033- \\
1,086\end{array}$ \\
\hline $\begin{array}{l}\text { Global RV } \\
\text { longitudinal } \\
\text { strain (\%) }\end{array}$ & 0.002 & 1.220 & $1.075-1.384$ & 0.001 & 1.152 & $\begin{array}{l}1.056- \\
1.257\end{array}$ & $<0.001$ & 1,158 & $\begin{array}{l}1,076- \\
1,245\end{array}$ \\
\hline $\begin{array}{l}\text { RV free wall } \\
\text { Longitudinal } \\
\text { strain (\%) }\end{array}$ & 0.02 & 1.048 & $1.0-1.099$ & 0.04 & 1.04 & $\begin{array}{l}1.002- \\
1.079\end{array}$ & 0.04 & 1.037 & $\begin{array}{l}1.005- \\
1.070\end{array}$ \\
\hline 2D GLS (\%) & $<0.001$ & 1,493 & 1.199-1,858 & $<0.001$ & 1.242 & $\begin{array}{l}1.116- \\
1.381\end{array}$ & $<0.001$ & 1,317 & $\begin{array}{l}1,184- \\
1,465\end{array}$ \\
\hline 2D GCS (\%) & 0.008 & 1,427 & $1.097-1.857$ & $<0.001$ & 1.447 & $\begin{array}{l}1.197- \\
1.750\end{array}$ & $<0.001$ & 1,329 & $\begin{array}{l}1,147- \\
1,539\end{array}$ \\
\hline 2D GRS (\%) & 0.98 & 1,001 & 0,932-1,075 & 0.38 & 0.975 & $\begin{array}{l}0.92- \\
1.032\end{array}$ & 0,92 & 0,998 & $\begin{array}{l}0,957- \\
1,041\end{array}$ \\
\hline $\begin{array}{l}3 \mathrm{D} \text { LA } \\
\text { indexed } \\
\text { volume }(\mathrm{ml})\end{array}$ & $<0.001$ & 1,075 & 1.033-1.118 & $<0.001$ & 1.058 & $\begin{array}{l}1.029- \\
1.088\end{array}$ & $<0.001$ & 1,054 & $\begin{array}{l}1,029- \\
1,079\end{array}$ \\
\hline \multicolumn{10}{|c|}{$\mathrm{HR}=$ hazard ratio; $\mathrm{Cl}=$ confidence interval). *Values of $\mathrm{p}<0.05$ indicate statistical significance. } \\
\hline $\begin{array}{l}\text { FAC, fractiona } \\
\text { LVDV, left ven } \\
\text { end-systolic } \\
\text { left ventricula } \\
\text { tricuspid ann }\end{array}$ & $\begin{array}{l}\text { Irea cha } \\
\text { cular di } \\
\text { ume; LV }\end{array}$ & $\begin{array}{l}\text { e; LA, I } \\
\text { olic vo }\end{array}$ & $\begin{array}{l}\text { atrium; LV, I } \\
\text { ne; LVEDV, le }\end{array}$ & ventric & $\begin{array}{l}\text { VDD, } \\
\text { end-d } \\
\text { /SD, le }\end{array}$ & $\begin{array}{l}\text { ventri } \\
\text { tolic v }\end{array}$ & $\begin{array}{l}\text { lar dias } \\
\text { me; LVE } \\
\text { r systol }\end{array}$ & $\begin{array}{l}\text { diam } \\
\text { left ve } \\
\text { iamete } \\
\text { dkness }\end{array}$ & $\begin{array}{l}\text { rr; } \\
\text { ricular } \\
\text { LVDV, } \\
\text { APSE, }\end{array}$ \\
\hline
\end{tabular}

$\mathrm{HR}=$ hazard ratio; $\mathrm{Cl}=$ confidence interval). *Values of $\mathrm{p}<0.05$ indicate statistical significance.

FAC, fractional area change; LA, left atrium; LV, left ventricle; LVDD, left ventricular diastolic diameter; LVDV, left ventricular diastolic volume; LVEDV, left ventricular end-diastolic volume; LVEDV, left ventricular end-systolic volume; LVEF, left ventricular ejection fraction; LVSD, left ventricular systolic diameter; LVDV, left ventricular diastolic diameter; RA, right atrium; RV, right ventricle; RWT, relative wall thickness; TAPSE, tricuspid annular plane systolic excursion.

\section{Death \\ Composite Endpoints}




\begin{tabular}{|c|c|c|c|c|c|c|c|c|c|}
\hline \multirow[b]{2}{*}{ 3D LVEF (\%) } & \multicolumn{3}{|c|}{$\begin{array}{l}\text { Hospitalization for Heart } \\
\text { Failure }\end{array}$} & \multicolumn{3}{|l|}{ Death } & \multicolumn{3}{|c|}{ Composite Endpoints } \\
\hline & 0.004 & 0,000 & $0.000-0.032$ & $<0.001$ & 0.000 & $\begin{array}{l}0.000- \\
0.008\end{array}$ & $<0.001$ & 0.000 & $\begin{array}{l}0,000- \\
0,002\end{array}$ \\
\hline 3D GLS (\%) & 0.012 & 1,146 & $1,03-1,275$ & $<0.001$ & 1.197 & $\begin{array}{l}1.090- \\
1.314\end{array}$ & $<0.001$ & 1,186 & $\begin{array}{l}1,102- \\
1,276\end{array}$ \\
\hline 3D GCS (\%) & 0.09 & 1,066 & $0,99-1,147$ & 0.005 & 1.076 & $\begin{array}{l}1.022- \\
1.132\end{array}$ & 0.01 & 1,062 & $\begin{array}{l}1,015- \\
1,112\end{array}$ \\
\hline $\begin{array}{l}\text { Area Strain } \\
(\%)\end{array}$ & 0.009 & 1,107 & $1,026-1,194$ & $<0.001$ & 1.155 & $\begin{array}{l}1.076- \\
1.239\end{array}$ & $<0.001$ & 1,123 & $\begin{array}{l}1,067- \\
1,083\end{array}$ \\
\hline 3D GRS & 0.009 & 0,933 & $0,885-0,992$ & $<0.001$ & 0.914 & $\begin{array}{l}0.871- \\
0.959\end{array}$ & $<0.001$ & 0,932 & $\begin{array}{l}0,901- \\
0,964\end{array}$ \\
\hline \multicolumn{10}{|c|}{$\mathrm{HR}=$ hazard ratio; $\mathrm{Cl}=$ confidence interval). ${ }^{*}$ Values of $\mathrm{p}<0.05$ indicate statistical significance. } \\
\hline \multicolumn{10}{|c|}{$\begin{array}{l}\text { FAC, fractional area change; LA, left atrium; LV, left ventricle; LVDD, left ventricular diastolic diameter; } \\
\text { LVDV, left ventricular diastolic volume; LVEDV, left ventricular end-diastolic volume; LVEDV, left ventricular } \\
\text { end-systolic volume; LVEF, left ventricular ejection fraction; LVSD, left ventricular systolic diameter; LVDV, } \\
\text { left ventricular diastolic diameter; RA, right atrium; RV, right ventricle; RWT, relative wall thickness; TAPSE, } \\
\text { tricuspid annular plane systolic excursion. }\end{array}$} \\
\hline
\end{tabular}

Death from all causes and Hospitalization for Heart Failure

As for composite endpoints, bilateral cardiac remodeling was associated with mortality. Larger atrial and ventricular diameters and volumes (absolute or indexed) as well as reduced LV and RV function (TAPSE) evaluated by 2D and 3D Echo, reduced velocities at tissue Doppler and increased E/e' ratio were associated with death from all causes and hospitalization for heart failure. Interestingly, RV S' wave, FAC were not associated with overall mortality.

2D LV GLS, 2D LV GCS, RV GLS, 3D LV GLS and 3D LV area strain were the strongest predictors of death from all causes and hospitalization for HF (Table 5).

ROC curves for prediction of cardiovascular outcomes

Receiver-operator characteristic (ROC) curves of the strong predictors of cardiovascular events were built for the five years of follow up (Figure 3). The cut offs of the STE parameters that yield the prediction of outcomes in five years of follow up are depicted in Table 6. 
Table 6

STE parameters' cut off values for prediction of events in five years of follow up.

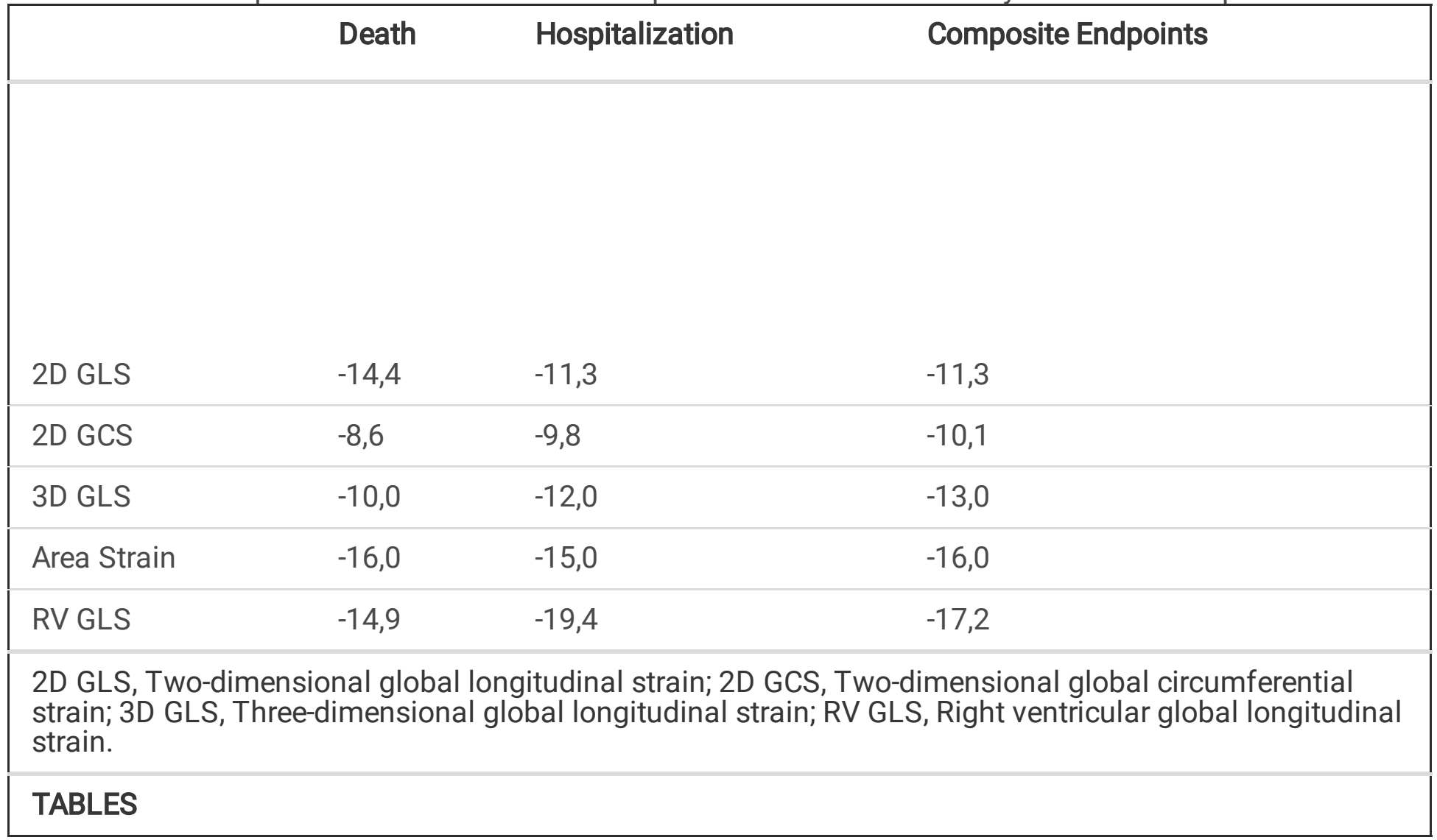

Table 6

STE parameter cutoff values for the prediction of events at five years of follow-up.

\begin{tabular}{|llll|}
\hline & Death & Hospitalization & Composite Endpoints \\
\hline 2D GLS & -14.4 & -11.3 & -11.3 \\
\hline 2D GCS & -8.6 & -9.8 & -10.1 \\
\hline 3D GLS & -10.0 & -12.0 & -13.0 \\
\hline Area Strain & -16.0 & -15.0 & -16.0 \\
\hline RV GLS & -14.9 & -19.4 & -17.2 \\
\hline $\begin{array}{l}\text { 2D GLS, two-dimensional global longitudinal strain; 2D GCS, two-dimensional global circumferential } \\
\text { strain; 3D GLS, three-dimensional global longitudinal strain; RV GLS, right ventricular global longitudinal } \\
\text { strain. }\end{array}$ \\
\hline
\end{tabular}

Reproducibility

The intraobserver and interobserver reproducibility of most of the STE parameters was excellent, as reflected by high CCC (Supplemental File; Table 7), with exception of 2D LV GCS and 2D LV GCS. Bland-Altman analysis demonstrated good intraobserver and interobserver agreement.

\section{Discussion}


To the best of our knowledge, this is the first study to evaluate 3D conventional and strain analysis in patients with CD from normal LVEF up to reduced LVEF. So far, only 2D STE parameters have been described in different stages of CD and most studies evaluated patients with normal LVEF ${ }^{18-21}$. Very few studies have reported 3D Echo conventional parameters in patients with CD but none have included 3D STE analysis ${ }^{22,23}$. The main findings of our study were: 1) 3D conventional and 2D STE analysis in mrLVEF were anatomically and functionally similar to rLVEF; 2) RV GLS, 2D LV GLS, 2D LV GCS, 3D LV GLS, and 3D LV area strain were strong predictors of outcomes in patients with $C D$, with superior value to other conventional parameters provided by cardiac dimensions, Tissue Doppler indexes or TAPSE and fractional area change for prediction of overall mortality or composite endpoints.

Our results are in disagreement with previous studies suggesting that in patients with the indeterminate form of $C D$ with pLVEF, global and segmental longitudinal systolic strain is reduced compared with healthy subjects, thus indicating that it could be a sensitive technique to detect early myocardial damage ${ }^{19,21}$. In other published investigation, only regional LV longitudinal strain was reduced in indeterminate form of CD with pLVEF that is in concordance with our results. Also, in a recent study, with patients in different CCM stages, 2D LV GLS was the more accurate measurement regarding stage A differentiation from the stages $B$, $C$, and $D^{24}$. However, reports of $2 D$ LV GLS in CD are conflicting and still remain a controversial issue. To explain the abnormal strain, myocarditis and inflammatory infiltrate was found in $15 \%$ and $37 \%$ respectively of indeterminate phase ${ }^{25}$. In another publication, in $60.6 \%$ of patients with indeterminate phase, it was found abnormalities like fiber degeneration, volume changes, interstitial edema, inflammatory infiltrates and fibrosis ${ }^{26}$. Besides, it was reported even in patients with Chagas disease with preserved or minimally impaired ventricular function cardiac fibrosis in $3 \%$ and $11 \%$ respectively using late gadolinium enhancement on cardiac magnetic resonance (CMR) ${ }^{27}$. Moreover, some studies support the use of 2D LV GLS in surveillance providing a window of opportunity for early intervention and preventing heart failure patients.

In our study, 3D conventional Echo and 2D STE parameters evidenced similar results comparing rLVEF and mrLVEF. Hitherto, the pathophysiology of rLVEF and mrLVEF heart failure patients is incompletely understood and, consequently, the reasons for this finding are unclear. In other etiologies, mrLVEF patients may include a heterogeneous population with patients that partially recovered the cardiac function under guidelinesdirected medical treatment, or patients that have not yet evolved to rLVEF, or patients that never will follow to rLVEF. Previously, we reported improvement in CCM under guidelines-directed medical treatment ${ }^{27}$. It was reported in reduced and mid-range LVEF HF patients similar natriuretic peptide elevated levels, and neuroendocrine profile. However, cardiac troponin values in mid-range LVEF HF patients are intermediate to those with reduced and preserved LVEF HF patients. On other hand, studies on endomyocardial biopsies from CD patients showed that the clinical evolution of the disease was correlated with a continuous progression of fiber destruction, fibrosis, myocardial inflammation, and a reduction in performance ${ }^{2}$. These findings may indicate that, possibly, patients with CD mrLVEF could be sharing with rLVEF some pathophysiological mechanisms related to 3D conventional Echo and 2D STE parameters but for all characteristics. In fact a recent meta-analysis concluded that significant differences in hospitalization and mortality were detected between mildly or mid-range heart failure and the other subtypes of heart failure including diverse etiologies but not included CD ${ }^{28}$. Nevertheless, CD is a very peculiar etiology that is not 
included in the studies. Herein, there is an extreme paucity of data concerning patients with mildly or midrange Chagas heart disease and no study so far evaluated 3D cardiac mechanics in patients with mrLVEF and $C D$.

Our findings that RV GLS, 2D LV GLS, 2D LV GCS, 3D LV GLS, and 3D LV area strain were strong predictors of outcomes in patients with $C D$ are in concordance with an increasing number of studies that have suggested that 2D LV GLS is superior to LVEF as a measure of LV function and as a predictor of mortality and cardiac events in other etiologies, mainly ischemic cardiac disease ${ }^{29}$. After adjustment for LVEF, there were no differences in STE values between Chagas Disease cardiomyopathy and idiopathic cardiomyopathy in reduced LVEF. 2D LV GLS was a strong predictor of adverse events, incremental to LVEF and E/e' ratio in dilated cardiomyopathy that included also chagasic etiology. No absolute values for 2D LV GLS indicating high risk are established, but a value of 2 D LV GLS of $-12 \%$ has been suggested representing severe systolic dysfunction and adverse prognosis ${ }^{29}$. Our findings are in concordance with this value and a value of 2D LV GLS lower than $-11.4 \%$ was associated with composite endpoints. 2D LV GCS is not well established as a predictor of outcomes because 2D LV GLS is the most robust parameter for this purpose but in our study, a value of 2D LV GCS lower than $-10.1 \%$ was also associated with composite endpoints.

The value of 2D LV GLS as a predictor of outcomes relies on the hypothesis that this parameter reflects changes in the myocardial interstitium yielding information regarding the extent of myocardial fibrosis as suggested by findings in patients with mitral regurgitation and hypertrophic cardiomyopathy. In another study, both GLS and ejection fraction were significant predictors of myocardial fibrosis. It was observed a high correlation with both ejection fraction assessed by echocardiography $(r=0.70, p<0.001)$ and GLS ( $r=$ $0.64, p<0.001)$ regarding the percentage of fibrosis. Nevertheless, after multiple linear regression analysis, the 2D LV GLS were no longer a predictor of myocardial fibrosis. So, the authors concluded in this study that 2D LV GLS has no incremental value to left ventricular ejection fraction assessed by conventional echocardiography in the prediction of myocardial fibrosis in patients with Chagas disease ${ }^{30}$.

In respect to the RV GLS, several studies showed that this parameter provides strong additional prognostic value to predict overall and cardiovascular mortality in rLVEF patients with other etiologies, essentially ischemic cardiac disease,. The predictive value was even higher than parameters evaluated by CMR as RV ejection fraction and RV strain ${ }^{31}$. In concordance in our study, RV GLS was also a strong predictor of outcomes. Values of RV GLS under $-17.2 \%$ and $-14.9 \%$ were associated with composite endpoints and mortality, respectively. Similarly, an RV GLS under $-19 \%$ was related to all-cause or cardiovascular mortality in rLVEF patients with ischemic, hypertensive or idiopathic cardiomyopathies ${ }^{31}$. As shown in previous studies in other etiologies and potentially also for CCM, RV GLS and RV free wall LS have performed better than conventional parameters for the prediction of outcomes. This may be probably because the use of the longitudinal strain for RV as for LV evaluation allows the analysis of the deformation of the endocardial fibers, which might be more sensitive to reduced coronary perfusion and increased wall stress and are usually affected earlier in myocardial diseases.

Concerning 3D analysis, our findings that 3D LV GLS and 3D LV area strain are strong predictors of outcomes in CCM suggest a very innovative clinical application for this technique. Until now, some studies 
evaluated the prognostic value of 3D Strain in a variety of clinical scenarios including valvular heart disease, ischemic heart disease and chronic renal failure, showing that the reduction of 3D values was associated with poor outcomes ${ }^{32}$. Particularly, 3D LV area strain is a very promising index that quantifies endocardial area change, integrating longitudinal and circumferential deformation, allowing a more detailed evaluation of the different types of myocardial fibers and enabling a better understanding of the pathophysiology of cardiomyopathies ${ }^{31,32}$. In a recent meta-analysis that aimed to determine normal ranges of 3D Strain, the authors reported that the mean value of 3D LV GLS was $19.1 \%$, ranging from 15.8 to $23.4 \%$ among the studies ${ }^{33}$. In our study, 3D LV GLS values and 3D LV area strain under $-13 \%$ and $-10 \%$, were associated with composite endpoints and mortality, respectively.

Clinical Implications

In our study, it was observed high feasibility of 2D and 3D strain analysis in all the groups. Despite the significant LV dilatation, especially in the group with rLVEF, which could limit the analysis of all LV segments, the feasibility was close to $100 \%$. These findings demonstrate the great applicability of this technique, even in patients with extensive ventricular remodeling and/or presence of abnormalities in the apical region, very frequent in patients with $\mathrm{CD}^{14}$.

The finding of better correlation of RV GLS, 2D LV GLS, 2D LV GCS, 3D LV GLS, and 3D LV area strain with outcomes than conventional 2D and 3D Echo conventional indexes in patients with CD provides important and potentially incremental information that may contribute to the establishment of cutoff values for different degrees of LV dysfunction and prognosis in addition to the already established values of LVEF. Therefore, we hypothesize that, eventually, the analysis of biventricular longitudinal strain could add incremental data regarding mortality and composite endpoints, predicting outcomes in patients with $C D$, as previously described, shedding light on complexes mechanisms of cardiomyocytes contraction imbalance and pathophysiology of patients with Chagas cardiomyopathy as a useful tool for prognostication.

Thereby, cardiac mechanics provide more refined and accurate information related to cardiac dysfunction and derangement than conventional parameters expressed by morphological variables as cardiac diameters and volumes and LVEF and might be related to myocardial fibrosis as evaluated by CMR but with expressive lower cost, mainly considering the economic issues in the developing countries, where CD is more prevalent.

\section{Limitations}

Due to the high prevalence of unknown or poorly controlled hypertension, in addition to diabetes mellitus and hypothyroidism, the vast majority of patients could not be included in this study, limiting the final number of patients. However, this extreme caution is mandatory when evaluating new techniques, without definite cutoff values and clinical applications. The small number of patients is a limitation as well as the fact that this was a single-center study.

Another limitations concern the limited availability of specific equipment besides more substantial time to acquire the datasets and perform all the measurements. Currently, conventional 3D Echo and 3D STE are available in a very limited number of research centers of echocardiography. Besides, both techniques require 
a learning curve and expertise until an adequate examination is possible. Nowadays, there is still inter-vendor variability in 3D STE measurements and spatial and temporal resolution are not yet adequate to perform reliable segmental analysis. Finally, there still considerable interobserver variability of 3D strain measurements but that was not a limitation in this study.

\section{Conclusions}

Patients with $C D$ and mrLVEF presented anatomic and functional characteristics more similar to the $C D$ and rLVEF despite the benign clinical evolution similar to patients with pLVEF. RV GLS, 2D LV GLS, 2D LV GCS, 3D LV GLS, and 3D LV area strain are strong predictors of outcomes in patients with CD. These findings suggest that 2D and 3D strain yields a potential incremental value for clinical decision guidance in patients with Chagas heart Disease.

\section{Abbreviations}

2D

Two-dimensional

3D

Three-dimensional

CD

Chagas Disease

GCS

global circumferential strain

GLS

global longitudinal strain

GRS

global radial strain

$\mathrm{HF}$

heart failure

LV

left ventricular

LVEF

left ventricular ejection fraction

RV

right ventricular

STE

speckle tracking echocardiography

TAPSE

Tricuspid annular plane systolic excursion

Echo

Echocardiography 


\section{Declarations}

\section{ACKNOWLEDGMENTS}

We acknowledge Mrs. Marcela Bergamini, from GE Healthcare Systems, for her support and help in providing ECHOPAC license.

\section{FUNDING}

There were no funding sources for this study.

\section{CONFLICT OF INTERESTS}

\section{All authors declare no conflict of interests. \\ PERSPECTIVES}

The use of new technologies for better understanding CD presents a great potential for clinical application. The evaluation of cardiac mechanics can be performed non-invasively, with practically no risk. Our study suggests that 2D and 3D strain analysis should be added to the comprehensive evaluation of patients with CD and rLVEF for prognostication. Our findings reinforce and extend previous studies' results showing that RV GLS, 2D, and 3D STE may provide additional information for better risk assessment of these patients with high feasibility and good intra and interobserver variability.

Although the application of strain imaging has yet not been included in clinical practice guidelines, it is likely to become a useful application when evaluating patients with CD.

\section{References}

1. Chagas, C. Nova tripanozomiaze humana. Estudos sob a morfologia e o ciclo evolutivo do Schizotrypanum cruz in. gen. s.sp. agente etiológico de nova entidade mórbida do homem. Mem Inst Osvaldo Cruz, 811, 1-95 (1909).

2. 2 et al. Chronic Chagas Heart Disease Management: From Etiology to Cardiomyopathy Treatment. J Am Coll Cardiol, 70 (12), 1510-1524 (2017).

3. 3 et al.J Am Coll Cardiol. The reality of heart failure in Latin America. 2013; 62(11):949-58

4. Torreão, J. A. et al. Myocardial tissue characterization in Chagas' heart disease by cardiovascular magnetic resonance.J Cardiovasc Magn Reson. 2015; 17:97

5. Andrade, J. P. et al. Sociedade Brasileira de Cardiologia. I Diretriz Latino Americana para o Diagnóstico e Tratamento da Cardiopatia Chagásica. Arq Bras Cardiol. 2011; 97(2 supl.3): 1-47

6. Mocelin, A. O. et al. The influence of aetiology on inflammatory and neurohumoral activation in patients with severe heart failure: a prospective study comparing Chagas' heart disease and idiopathic dilated cardiomyopathy. Eur J Heart Fail, 7 (5), 869-73 (2005). 
7. Pereira Nunes Mdo, C. et al. Predictors of mortality in patients with dilated cardiomyopathy: relevance of Chagas disease as an etiological factor. Rev Esp Cardiol, 63, 788-97 (2010).

8. Rassi, A. Jr, Rassi, A. \& Rassi, S. G. Predictors of mortality in chronic Chagas disease: a systematic review of observational studies., 115, 1101-8 (2007).

9. Rassi, R. J. A. \& Little, A. Development and Validation of a Risk Score for Predicting Death in Chagas' Heart Disease. N Engl J Med, 355, 799-808 (2006).

10. Garzon, S. A. C. et al. Predictors of mortality in chronic Chagas heart disease long-term follow-up of 987 subjects for up to 22 years.J Am Coll Cardiol. 1998; 31(suppl C):107C.

11. Viotti, R. J. et al. Value of echocardiography for diagnosis and prognosis of chronic Chagas disease cardiomyopathy without heart failure., 90, 655-660 (2004).

12. Nunes, M. C. et al. Right ventricular dysfunction is an independent predictor of survival in patients with dilated chronic Chagas cardiomyopathy. Int J Cardiol, 127, 372-9 (2008).

13. Nunes, M. C. P. et al. Multimodality imaging evaluation of Chagas Disease: an expert consensus of Brazilian Cardiovascular Imaging Department (DIC) and the European Association of Cardiovascular Imaging (EACVI). Eur Heart J Cardiovasc Imaging, 19 (4), 459-460 (2018).

14. Acquatella, H. et al. Recommendations for Multimodality Cardiac Imaging in Patients with Chagas Disease: A Report from the American Society of Echocardiography in Collaboration With the InterAmerican Association of Echocardiography (ECOSIAC) and the Cardiovascular Imaging Department of the Brazilian Society of Cardiology (DIC-SBC). J Am Soc Echocardiogr, 31 (1), 3-25 (2018).

15. Amundsen, B. H. et al. Noninvasive myocardial strain measurement by speckle tracking echocardiography: validation against sonomicrometry and tagged magnetic resonance imaging. J Am Coll Cardiol, 47, 789-93 (2006).

16. Mizuguchi, Y. et al. The functional role of longitudinal, circumferential, and radial myocardial deformation for regulating the early impairment of left ventricular contraction and relaxation in patients with cardiovascular risk factors: a study with two-dimensional strain imaging. J Am Soc Echocardiogr, 21 (10), 1138-44 (2008).

17. Lang, R. M. et al. Recommendations for chamber quantification by echocardiography in adults: an update from the American Society of Echocardiography and the European Association of Cardiovascular Imaging. J Am Soc Echocardiogr, 28 (1), 1-3914 (2015).

18. Garcia-Alvarez, A. et al. Myocardial deformation analysis in Chagas heart disease with the use of speckle tracking echocardiography. J Card Fail, 17, 1028-34 (2011).

19. Gomes, V. A. et al. Analysis of Regional Left Ventricular Strain in Patients with Chagas Disease and Normal Left Ventricular Systolic Function. J Am Soc Echocardiogr, 29 (7), 679-88 (2016).

20. Barros, M. V. et al. Mechanical dispersion assessed by strain echocardiography is associated with malignant arrhythmias in Chagas cardiomyopathy. J Am Soc Echocardiogr, 29, 368-74 (2016).

21. Romano, M. M. D. et al. Early impairment of myocardial deformation assessed by regional speckletracking echocardiography in the indeterminate form of Chagas disease without fibrosis detected by cardiac magnetic resonance. J Am Soc Echocardiogr, 29 (7), 679-88 (2016). 
22. Hotta, V. T. et al. Rare association of endomyocardial fibrosis and Chagas heart disease. Eur Heart $J$ Cardiovasc Imaging, 18 (3), 378-379 (2017).

23. Saraiva, R. M. et al. Left Atrial Structure and Function Predictors of New-Onset Atrial Fibrillation in Patients with Chagas Disease. J Am Soc Echocardiogr. 2020;33(11):1363-1374. 24.,, Echocardiographic parameters, speckle tracking, and brain natriuretic peptide levels as indicators of progression of indeterminate stage to Chagas cardiomyopathy. Echocardiography. 2020;37(3):429-438

24. Mady, C., Pereira-Barretto, A. C., lanni, B. M., Lopes, E. A. \& Pileggi, F. Right ventricular endomyocardial biopsy in undetermined form of Chagas' disease., 35, 755-9 (1984).

25. Higuchi, M. L. Endomyocardial biopsy in Chagas' heart disease: pathogenetic contributions.Sao Paulo Med. J.195;113.

26. Tassi, E. M., Continentino, M. A., Nascimento, E. M., Pereira, B. B. \& Pedrosa, R. C. Relationship between fibrosis and ventricular arrhythmias in Chagas heart disease without ventricular dysfunction. Arq Bras Cardiol, 102 (5), 456-64 (2014).

27. Issa, V. S. et al. Beta-blocker therapy and mortality of patients with Chagas cardiomyopathy: a subanalysis of the REMADHE prospective trial. Circ Heart Fail, 3, 82-8 (2010).

28. Altaie, S. \& Khalife, W. The prognosis of mid-range ejection fraction heart failure: a systematic review and meta-analysis. ESC Heart Failure, 5, 1008-1016 (2018).

29. Ersboll Mads, Valeur, N. et al. Prediction of all-cause mortality and heart failure admissions from global left ventricular longitudinal strain in patients with acute myocardial infarction and preserved left ventricular ejection fraction. J Am Coll Cardiol, 61 (23), 2365-73 (2013).

30. Macedo, C. T. et al. Assessment of speckle tracking strain predictive value for myocardial fibrosis in subjects with Chagas disease. Int J Cardiol Heart Vasc, 8, 75-80 (2015).

31. Houard, L. et al. Additional Prognostic Value of 2D Right Ventricular Speckle-Tracking Strain for Prediction of Survival in Heart Failure and Reduced Ejection Fraction: A Comparative Study With Cardiac Magnetic Resonance.J Am Coll Cardiol Img2019; 12:2373-85

32. Medvedofsky, D. et al. 2D and 3D echocardiography-derived indices of left ventricular function and shape: relationship with mortality. J Am Coll Cardiol Img, 11 (11), 1569-79 (2018).

33. Truong, V. T. et al. Normal Ranges of Left Ventricular Strain by Three-Dimensional Speckle Tracking Echocardiography in Adults: A Systematic Review and Meta-Analysis. J Am Soc Echocardiogr, 32 (12), 1586-975 (2019).

\section{Figures}



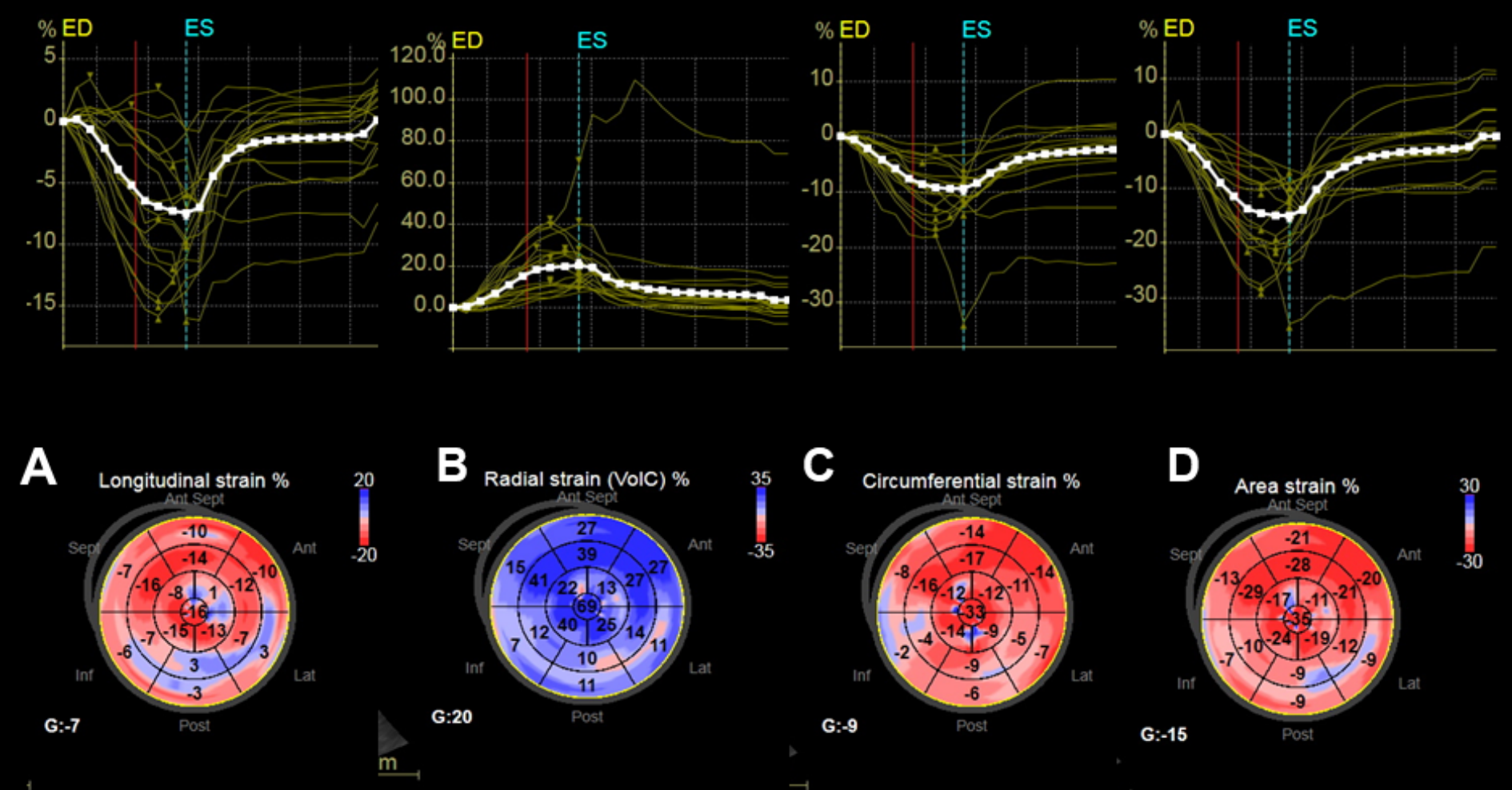

\section{Figure 1}

3D STE images from a patient with CD cardiomyopathy and severe LV dysfunction. A, 3D LV global longitudinal strain $=-7 \%$. B, 3D LV global radial strain $=20 \%$. C, 3D LV global circumferential strain $=-9 \%$. 3D LV area strain $=-15 \%$. 3D STE parameters are reduced diffusely. 3D STE, Three-dimensional speckle tracking echocardiography; CD, Chagas disease; LV, left ventricular. 

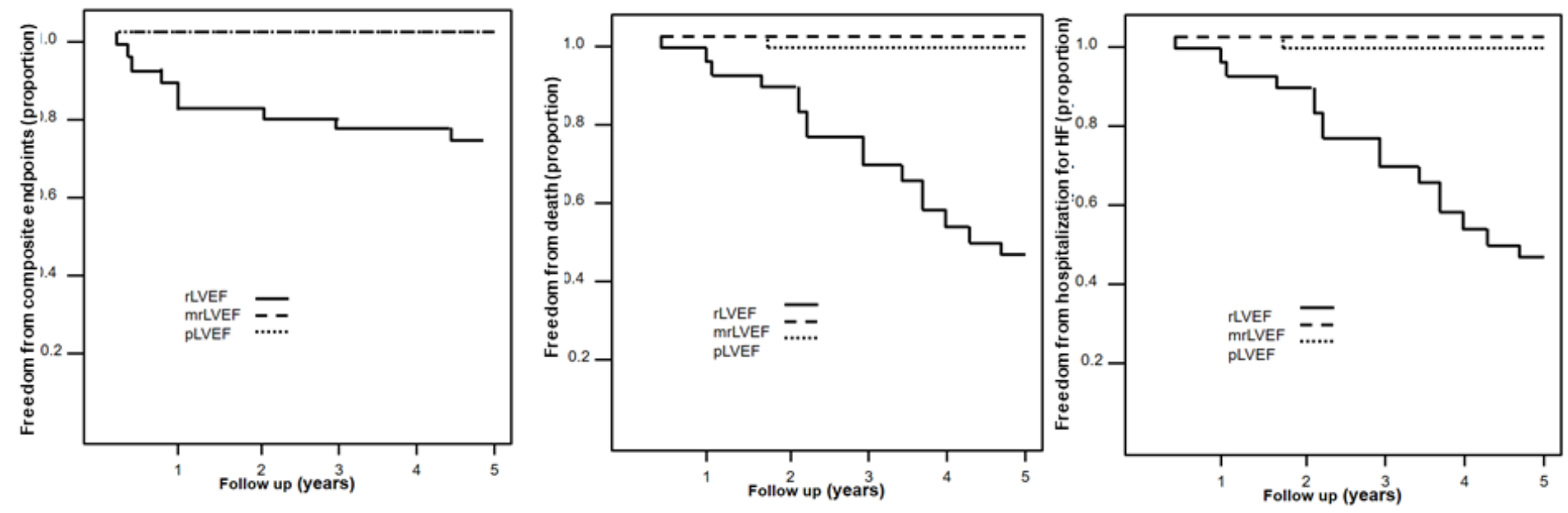

Figure 2

Composite endpoints (hospitalization for HF, complex ventricular arrhythmias, heart transplant and death), survival and hospitalization for heart failure Kaplan Meyer curves are shown for the three groups. HF, heart failure; rLVEF, reduced left ventricular ejection fraction; mrLVEF, mid-range or mildly ejection fraction; pLVEF, preserved left ventricular ejection fraction. 
2D LV GLS
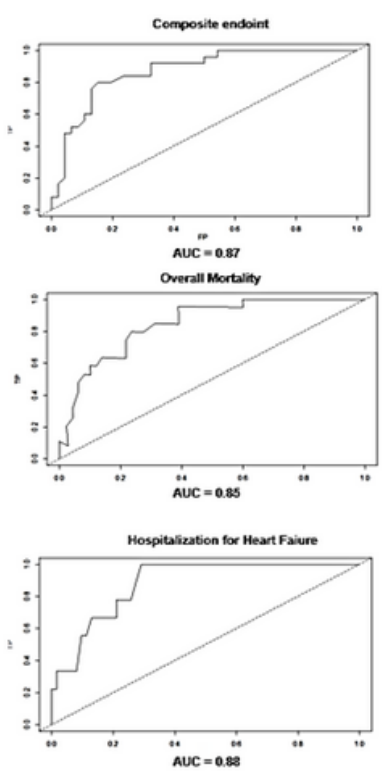

2D LV GCS
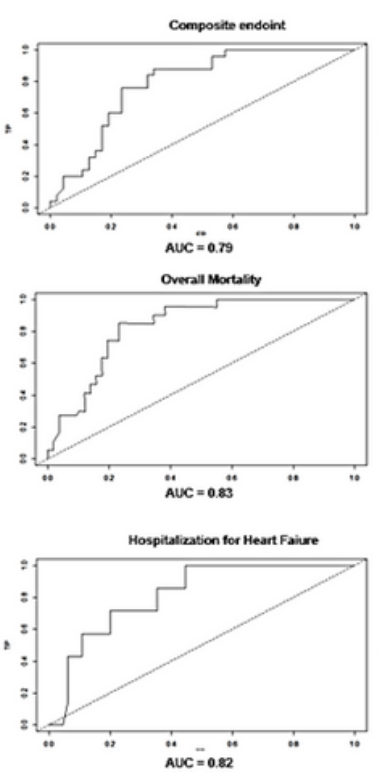

3D LV GLS
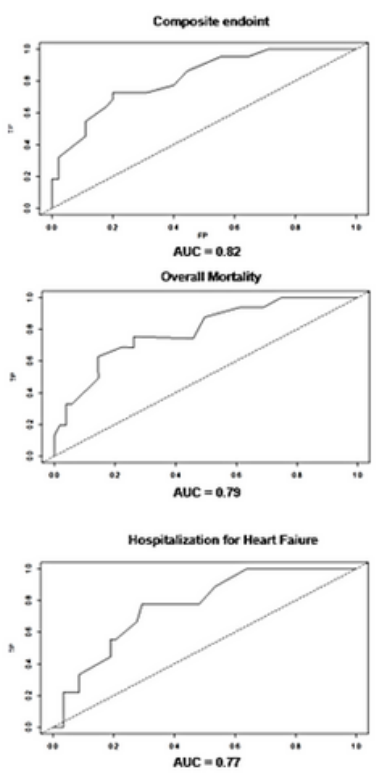

3D LV Area strain
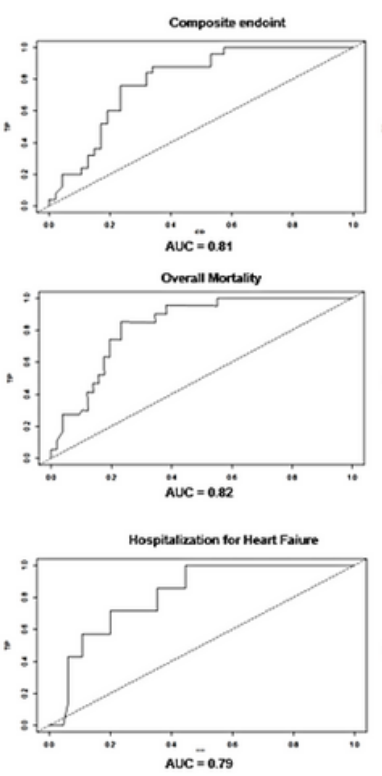

RV GLS
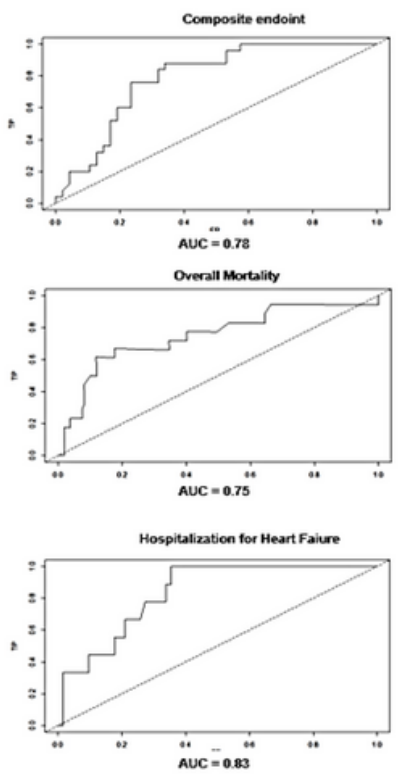

\section{Figure 3}

Receiver-operator characteristic (ROC) curves of the strong predictors of cardiovascular events during the five years of follow up. 2D LV GLS, Two-dimensional left ventricular global longitudinal strain; 2D LV GCS, Twodimensional left ventricular global circumferential strain; 3D LV GLS, Three-dimensional left ventricular global longitudinal strain; 3D LV Area strain, Three-dimensional left ventricular area strain; AUC, area under curve; RV GLS, Right ventricular global longitudinal strain.

\section{Supplementary Files}

This is a list of supplementary files associated with this preprint. Click to download.

- SUPPLEMENTALFILES.docx 